\title{
Illumination Invariant Face Recognition Using Fuzzy LDA and FFNN
}

\author{
Behzad Bozorgtabar, Hamed Azami, Farzad Noorian
}

School of Electrical Engineering, Iran University of Science and Technology, Tehran, Iran.

Email: b_bozorgtabar@elec.iust.ac.ir, hmdazami@gmail.com, fnoorian@ee.iust.ac.ir

Received October $20^{\text {th }}, 2011$; revised November $24^{\text {th }}, 2011$; accepted December $10^{\text {th }}, 2011$

\begin{abstract}
The most significant practical challenge for face recognition is perhaps variability in lighting intensity. In this paper, we developed a face recognition which is insensitive to large variation in illumination. Normalization step including two steps, first we used Histogram truncation as a pre-processing step and then we implemented Homomorphic filter. The main idea is that, achieving illumination invariance causes to simplify feature extraction module and increases recognition rate. Then we utilized Fuzzy Linear Discriminant Analysis (FLDA) in feature extraction stage which showed a good discriminating ability compared to other methods while classification is performed using Feedforward Neural Network (FFNN). The experiments were performed on the ORL (Olivetti Research Laboratory) face image database and the results show the present method outweighs other techniques applied on the same database and reported in literature.
\end{abstract}

Keywords: Face Recognition; Histogram Truncation; Homomorphic Filter; Fuzzy LDA; FFNN

\section{Introduction}

Face recognition has become one of the most active research areas of pattern recognition since the early 1990s, and has attracted substantial research efforts from the areas of computer vision, bio-informatics and machine learning.

Illumination is considered one of the most difficult tasks for face recognition. The illumination setup in which recognition is performed is in most cases impractical to control, its physics difficult to accurately model and face appearance differences due to changing illumination are often larger than those differences between individuals. Reliable techniques for recognition under more extreme variations caused by pose, expression, occlusion or illumination is highly nonlinear, have proven elusive [1].

In this paper, we outline a hybrid technique for illumination normalization, after the Histogram truncation was applied to input face images, the Homomorphic filter was used for normalization. Then face recognition is performed with two major steps. In the first step, some useful features of the image are extracted. In the second step, on the basis of the extracted features the classification is executed.

Fuzzy LDA (Fuzzy Fisherface) recently, was proposed for feature extraction and face recognition [2]. Fuzzy LDA computes fuzzy within-class scatter matrix and between- class scatter matrix by incorporating class membership of the binary labeled faces (patterns).

Finally extracted features were considered as inputs to classifiers. In this paper well-known classifier, Feedforward Neural Networks were employed as classification.

Then rest of this paper is as followed. The preprocesssing step is initiated in Section 2 then Fuzzy LDA is introduced in Section 3. Section 4 describes classification and Section 5 determines our experimental results on ORL dataset, and Conclusions is given in Section 6.

\section{Illumination Normalization Technique}

In this stage, in order to boost the result of normalization, we first truncated a specified percentage of the lower and upper ends of an image histogram.

In fact, several studies have shown that histogram remapping in conjunction with photometric normalization techniques results in better face recognition performance than using photometric normalization techniques on their own.

In the next step, Homomorphic filter as a renowned illumination reflectance was used. And then filtered face image is considered as input of feature extraction module.

\section{Homomorphic Filters}

Homomorphic filtering (HOMO) is a well known norma- 
lization technique, which improves the appearance of an image by contrast enhancement and gray-level range compression.

Consider an image, $f(x, y)$, which can be stated as the product of the illumination $i(x, y)$, and the reflectance component $r(x, y)$ as follows [3]:

$$
f(x, y)=i(x, y) \cdot r(x, y)
$$

Then input image is transformed in to the logarithm domain in order to achieve frequency components of the illumination and reflectance separately:

$$
\begin{aligned}
z(x, y) & =\ln f(x, y) \\
& =\ln i(x, y)+\ln r(x, y)
\end{aligned}
$$

Then:

Or:

$$
\begin{aligned}
\mathfrak{F}\{z(x, y)\} & =\mathfrak{F}\{\ln f(x, y)\} \\
& =\mathfrak{F}\{\ln i(x, y)\}+\mathfrak{F}\{\ln r(x, y)\}
\end{aligned}
$$

$$
Z(u, v)=F_{i}(u, v)+F_{r}(u, v)
$$

where $F_{i}(u, v)$ and $F_{r}(u, v)$, in Equation (2) are the Fourier transforms of the term defined.

The Fourier transform of the product of the $Z(u, v)$ and filter function $H(u, v)$ can be expressed as:

$$
\begin{aligned}
S(u, v) & =H(u, v) \cdot Z(u, v) \\
& =H(u, v) \cdot F_{i}(u, v)+H(u, v) \cdot F_{r}(u, v)
\end{aligned}
$$

In the spatial domain:

$$
\begin{aligned}
s(x, y) & =\mathfrak{F}^{-1}\{S(u, v)\} \\
& =\mathfrak{F}^{-1}\left\{H(u, v) . F_{i}(u, v)+H(u, v) . F_{r}(u, v)\right\}
\end{aligned}
$$

Finally by letting

$$
\begin{aligned}
& i(x, y)=\mathfrak{F}^{-1}\left\{H(u, v) . F_{i}(u, v)\right\} \\
& r(x, y)=\mathfrak{F}^{-1}\left\{H(u, v) . F_{r}(u, v)\right\}
\end{aligned}
$$

The equation becomes:

$$
\begin{gathered}
s(x, y)=i(x, y)+r(x, y) \\
g(x, y)=e^{s(x, y)} \\
g(x, y)=e^{i(x, y)}+e^{r(x, y)} \\
g(x, y)=i_{0}(x, y)+r_{0}(x, y)
\end{gathered}
$$

where $i_{0}$ and $r_{0}$ are the illumination and the reflectance components of the output images. After $z(x, y)$ is transformed into the frequency domain, the high frequency components are emphasized and the low-frequency components are reduced. As a final step the image is transformed back into the spatial domain by applying the inverse Fourier transform and taking the exponential of the result.

This method is based on a special case of a class of systems known as Homomorphic system. The filter trans- form function $H(u, v)$ is known as the Homomorphic filter [4] .

\section{Fuzzy LDA (FLDA)}

In Fuzzy LDA, which also was called Fuzzy Fisher Face method, the basic LDA is changed. The modification is the introduction of fuzziness in to the belonging of projected vector to the classes which solves binary classification problems. In conventional LDA approach, every vector is supposed to have a crisp membership. But this does not take into account the resemblance of images belonging to different classes, which occurs under varying conditions. In FLDA, each vector is assigned the membership grades of every class based upon the class label of its $k$ nearest neighbors. This Fuzzy $k$-nearest neighbor is utilized to evaluate the membership grades of all the vectors [5].

$u_{i j}$ stands for the membership grade of $j^{\text {th }}$ vector in the $i^{\text {th }}$ class and satisfies two obvious properties:

$$
\begin{array}{r}
\sum_{i=1}^{C} u_{i j}=1 \\
0<\sum_{j=1}^{N} u_{i j}<N
\end{array}
$$

The class labels of the $k$ vectors located in the closest neighborhood of each vector is collected during the training phase. Then the membership grade of $j^{\text {th }}$ vector in the $i^{\text {th }}$ class is evaluated as follow [5]:

$$
u_{i j}=\left\{\begin{array}{cc}
0.51+0.49\left(n_{i j} / k\right) & i, j \text { belong to the same class } \\
0.49\left(n_{i j} / k\right) & \text { otherwise }
\end{array}\right.
$$

In the above expression $n_{i j}$ stands for the number of the neighbors of the $j^{\text {th }}$ data (pattern) that belong to the $i^{\text {th }}$ class.

The moderated membership grades are used in the computations of the statistical properties of the patterns. The mean vector of each class is obtained from the below equation [6]:

$$
m_{i}=\frac{\sum_{j=1}^{N} u_{i j} x_{j}}{\sum_{j=1}^{N} u_{i j}}
$$

Then, the corresponding fuzzy within-class scatter matrix and fuzzy between-class scatter matrix can be redefined as follow $[7,8]$ :

$$
\begin{gathered}
F S_{W}=\sum_{i=1}^{c} \sum_{x_{j} \in w_{i}} u_{i j}\left(x_{j}-m_{i}\right)\left(x_{j}-m_{i}\right)^{T} \\
F S_{b}=\sum_{i=1}^{c} \sum_{j=1_{i}}^{N} u_{i j}\left(m_{i}-\bar{X}\right)\left(m_{i}-\bar{X}\right)^{T}
\end{gathered}
$$


where $\bar{X}$ is the mean of all samples. Our optimal fuzzy projection $W_{F-L D A}$ follows the expression:

$$
W_{F-L D A}=\arg \max _{w} \frac{\left|W^{T} F S_{B} W\right|}{\left|W^{T} F S_{W} W\right|}
$$

It is difficult to directly calculate $W_{F-L D A}$ because that $F S_{W}$ is often singular [6].

For tackle this problem, PCA is used as a dimension reduction step and thus the final transformation is given by the following matrix,

$$
W^{T}=W_{F-L D A}^{\mathrm{T}} W_{P C A}^{\mathrm{T}}
$$

\section{Classification}

\section{Feedforward Neural Networks}

Neural networks have been a much-publicized topic of research in recent years and are now beginning to be used in a wide range of subject areas. Having high computation rates, neural network is used as classification. For this purpose each input pattern is forced, adaptively, to output the pattern indicators that are part of the training data. Feed-forward networks, sometimes called multilayer perceptrons (MLP), are trained adaptively to transform a set of input signals, $X$, into a set of output signals, $O[9,10]$.

Feedback networks start with an initial activity state of a feedback system, and after state transitions have taken place, the asymptotic final state is identified as the outcome of the computation [11].

Figure 1 shows the architecture of the system for face classification. After calculating the features, the feature projection vectors are calculated for the faces in the database. These feature projection vectors are used as inputs to train the neural network. Figure 2 illustrates the schematic diagram for the training phase. In test phase, image's feature projection vector is calculated from the feature space. These vectors are fed to the neural network and the network is simulated by them, where the network outputs are compared. The new face's class is determined by considering maximum output of different classes in which the class with maximum output clarify test image's label.

The two activation functions $f_{h}$ (input layer to hidden layer) and $f_{o}$ (hidden layer to output layer) are used which are logistic functions:

$$
\begin{aligned}
& f_{h}=\frac{1}{1+\exp \left(-\lambda_{h}\right)} \\
& f_{o}=\frac{1}{1+\exp \left(-\lambda_{o}\right)}
\end{aligned}
$$

$\lambda_{h}$ and $\lambda_{o}$ are inputs for hidden layer and output layer respectively.

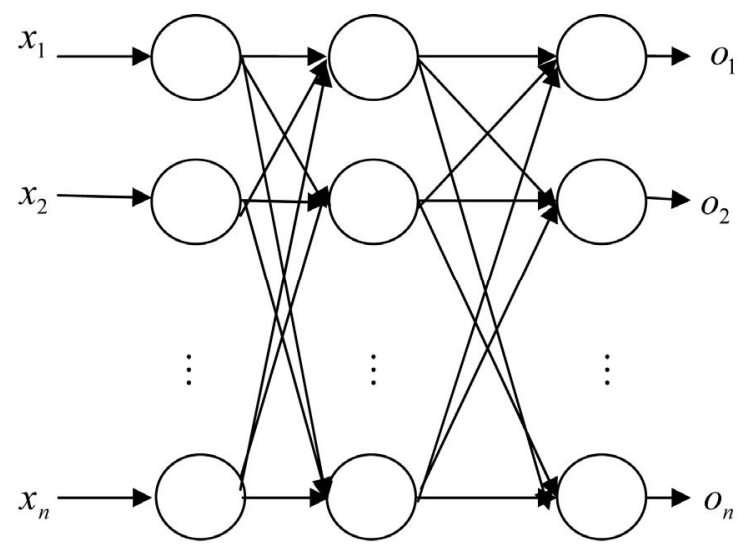

Figure 1. Architecture of FFNN for classification.

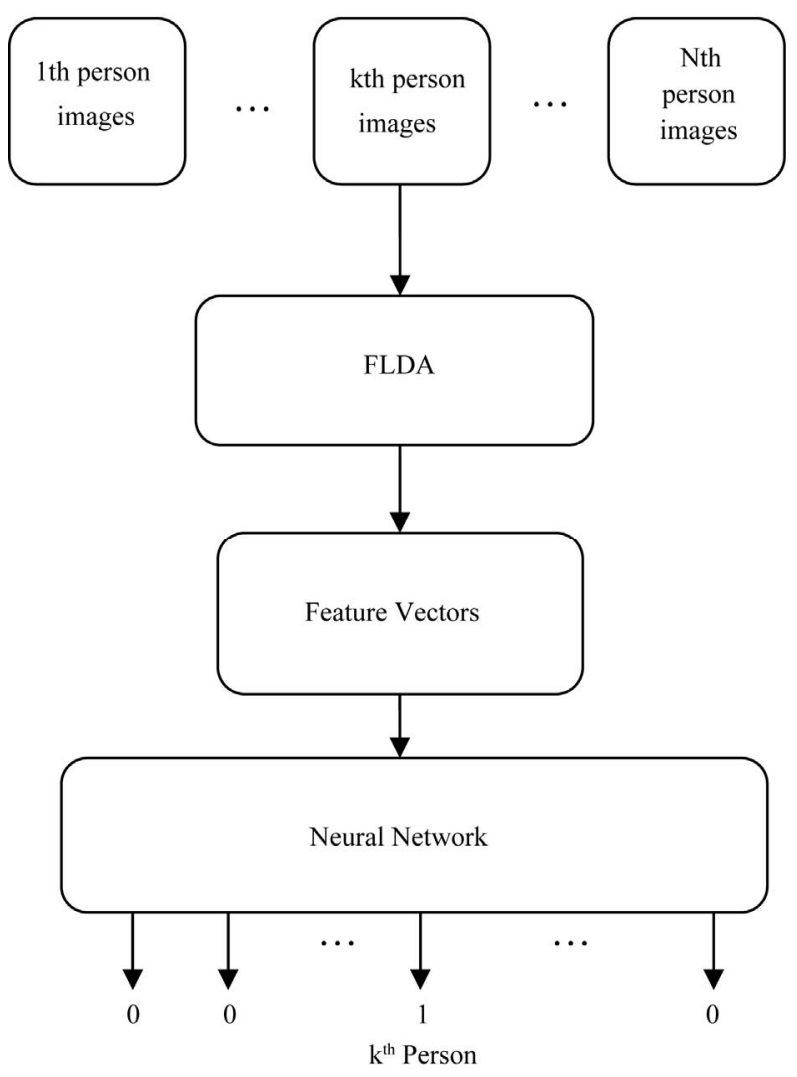

Figure 2. Training stage for neural network.

\section{Implementation and Results}

The ORL database consists of 40 groups [12], each containing ten $112 \times 92$ gray scale images of a single subject. Each subject's images differ in lighting, facial expression, details (i.e. glasses/no glasses) and even sliding. Some of the database's images are illustrated in Figure 3.

\subsection{Preprocessing Step}

Figure 4 shows an original image from ORL database and its histogram respectively. This image is chosen spe- 


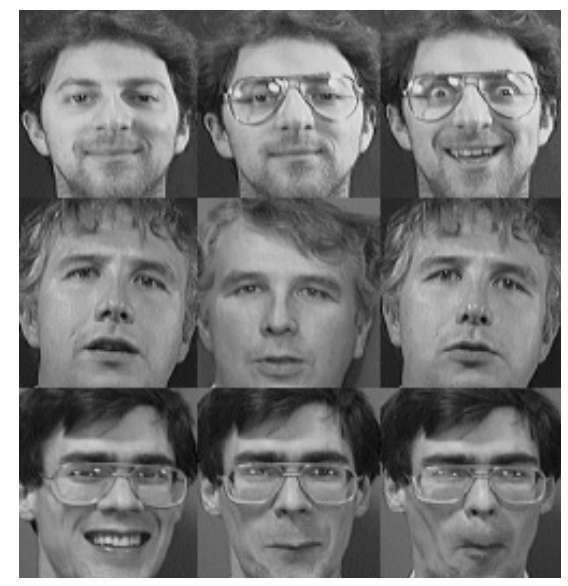

Figure 3. Samples of ORL face database.
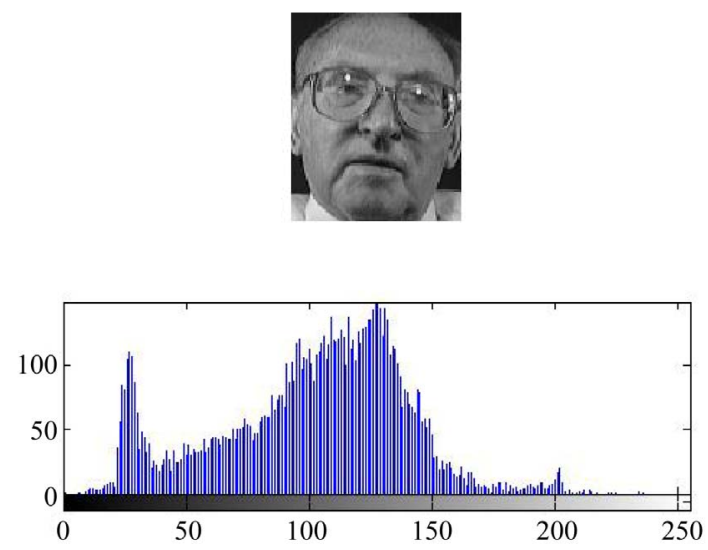

Figure 4. Original image and the corresponding histogram.

cifically because it had led to misclassification in many feature extraction methods. Being taken under ambient lighting in a neutral facial expression and the person wore glasses, the images of this class lead to increase in error rates. In the next step, the hybrid method (Histogram truncation-Homomorphic filter) was applied to the selected image for normalization. The filtered image and its histogram are displayed by Figure 5. Initially we implemented the Histogram truncation, in this step the lower and upper ends of an image histogram that must be truncated, were set to 20 percent and 60 percent respectively.

Then Homomorphic filter was used, the performance of this filter rely on its parameters. We set the cut-off frequency of the filter to 0.5 and second order of the modified Butterworth style filter is used. In the next step, we shortened half of upper ends of final histogram.

\subsection{Feature Extraction Module}

After preprocessing step, FLDA was applied in order to extract features. The experiment was performed using the first ( $l=3,4$ and 5 ) images per class for training, and the remaining images for testing. Figure 6 demonstrates com-
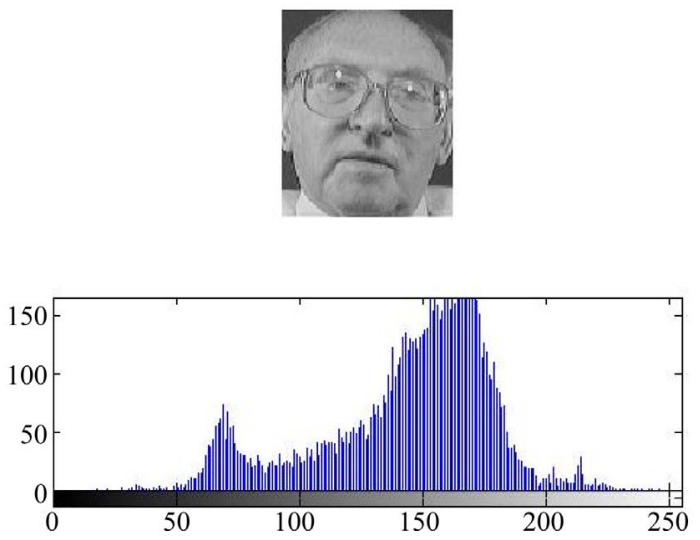

Figure 5. Filtered image and the corresponding histogram.

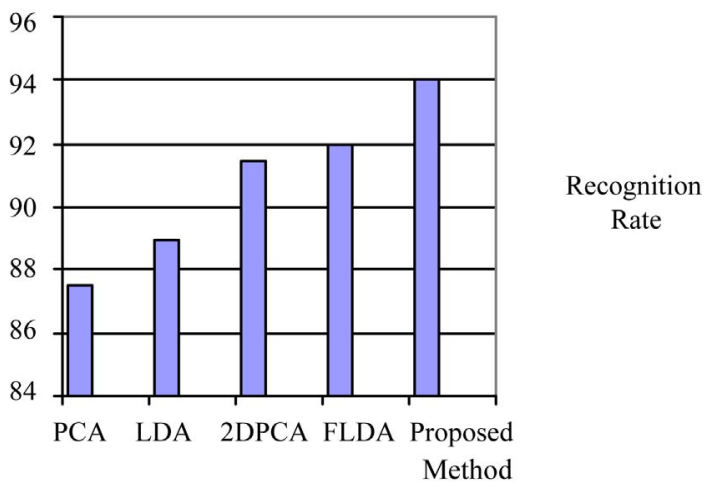

Figure 6. Comparison of recognition rates of proposed Feature extractors based on nearest neighbor classifier for ORL face database.

parison between different feature extractors with Nearest Neighbor classifier which the number of training images is 4 .

For two images $i$ and $j$, let $f^{(i)}$ and $f^{(j)}$ representing the corresponding feature vectors, the distance $d_{i j}$ between the two patterns in the feature space is defined as:

$$
d_{i j}=\sqrt{\sum_{n}\left(\frac{f_{(n)}^{(i)}-f_{(n)}^{(j)}}{\alpha\left(f_{n}\right)}\right)^{2}}
$$

where $f_{n}^{(i)}$ is the $n$th element of the feature vector $i$ while the term $\alpha\left(f_{n}\right)$ is the standard deviation of the $n$th element over the entire database and is used to normalize the individual feature components. Finally, a test image $j$ is assigned to image $i$ in a database with the smallest corresponding distance $d_{i j}$.

Feature extraction, which were used, PCA (eigenface), LDA (Fisherface), 2D-PCA, Conventional Fuzzy fisherface without normalization step and the proposed method, respectively. In the PCA phase of PCA, LDA and Fuzzy Fishface, we keep nearly 98 percent image energy and the nearest neighborhood parameter $K$ in Fuzzy Fisherface and our proposed technique was set to $k=3$. 


\subsection{Classification Phase}

Feedforward Neural Network employs Backpropagation for learning process of network which in there the output vector $o$ of each input vector is compared with target vector $t$.

Weights of network are updated every iteration in order to minimize the cost function or the mean square error between the output vector and the target vector; this is obtained from below equation:

$$
E=\frac{1}{2} \sum_{i=1}^{k}\left(o_{i}-t_{i}\right)^{2}
$$

$K$ is the number of neurons in output layer. In this experiment the number of nodes in hidden layer is set to 15 . Two set of images were created from the ORL database; For the Five-to-Five dataset, five random images of each group were selected for training while the others were used for testing. For the Leave-One-Out set, 9 images were used for training and the remaining image was kept for validation.

Table 1 shows comparison of recognition rates of various methods in Five-to Five set while Table 2 illustrates figures which compare our proposed method with conventional Fuzzy LDA without normalization step based on the number of training samples. As we see from the table, with increasing in the number of training samples, the recognition rates will grow and our method outweigh conventional FLDA.

Table 3 demonstrates recognition rates of depicted techniques with FFNN classifier for two sets.

Table 1. Comparison of recognition rates of different classifiers based on various feature extractors for ORL face database.

\begin{tabular}{ccc}
\hline & Nearest Neighbor & FFNN \\
\hline PCA & $88 \%$ & $89 \%$ \\
LDA & $90 \%$ & $91 \%$ \\
2D-PCA & $93 \%$ & $93 \%$ \\
Proposed & $95 \%$ & $95.5 \%$ \\
\hline
\end{tabular}

Table 2. Comparison between recognition rates of conventional FLDA and our proposed method based on two classifiers (nearest neighbor, feedforward $\mathrm{NN}$ ) in terms of different training samples.

\begin{tabular}{cccccc}
\hline $\begin{array}{c}\text { Training } \\
\text { images }\end{array}$ & $\begin{array}{c}\text { Test } \\
\text { images }\end{array}$ & $\begin{array}{c}\text { Proposed } \\
\text { method } \\
+ \text { FFNN }\end{array}$ & $\begin{array}{c}\text { Proposed } \\
\text { method } \\
+ \text { NN }\end{array}$ & $\begin{array}{c}\text { Conventional } \\
\text { method } \\
+ \text { FFNN }\end{array}$ & $\begin{array}{c}\text { Conventional } \\
\text { method } \\
+ \text { NN }\end{array}$ \\
\hline 3 & 7 & $91 \%$ & $91.5 \%$ & $90.5 \%$ & $91 \%$ \\
4 & 6 & $95 \%$ & $94 \%$ & $92.5 \%$ & $92 \%$ \\
5 & 5 & $95.5 \%$ & $95 \%$ & $94.5 \%$ & $94 \%$ \\
\hline
\end{tabular}

Table 3. Comparison of recognition rates of different methods for two sets.

\begin{tabular}{ccc}
\hline & Five-to-Five & Leave-One-Out \\
\hline PCA & $89 \%$ & $89.5 \%$ \\
LDA & $91 \%$ & $91.5 \%$ \\
2D-PCA & $93 \%$ & $94.5 \%$ \\
Proposed & $95.5 \%$ & $96.5 \%$ \\
\hline
\end{tabular}

\section{Conclusions}

In this paper, the Face Recognition problem was addressed by improved method based on modified Homomorphic filter, which is insensitive to large variation in illumination. Homomorphic filter is a celebrated normalization technique which was ignored in face recognition. In continue Fuzzy LDA (FLDA) in collaboration with depicted preprocessing step showed a good ability in order to extract useful features which led to increase in recognition rate for different classifiers.

Finally, Feedforward Neural Network, was used which the result showed proposed method with this classifier had a better performance compared with Nearest Neighbor classifier.

\section{REFERENCES}

[1] Y. A. Georghiades, P. Belhumeur and D. Kriegman, "From Few to Many: Illumination Cone Models for Face Recognition under Variable Lighting and Pose,” IEEE Transactions on Pattern Analysis and Machine Intelligence, Vol. 23, No. 6, 2001, pp. 643-660. doi:10.1109/34.927464

[2] K. C. Kwak and W. Pedrycz, "Face Recognition Using a Fuzzy Fisher Classifier,” Pattern Recognition, Vol. 38, No. 10, 2005, pp. 1717-1732. doi:10.1016/j.patcog.2005.01.018

[3] A. M. E. Thammizharasi, "Performance Analysis of Face Recognition by Combining Multiscale Techniques and Homomorphic Filter Using Fuzzy k-Nearest Neighbour Classifier," IEEE International Conference on Communication Control and Computing Technologies, Ramanathapuram, 7-9 October 2010, pp. 643-650.

[4] N. A. Surobhi and Md. R. Amin, "Employment of Modified Homomorphic Filters in Medical Imaging," International University Journal of Science and Technology in Daffodil, Vol. 1, No. 1, 2006.

[5] J. M. Keller, M. R. Gray and J. A. Givern, "A Fuzzy K Nearest Neighbor Classifier Algorithm,” IEEE Transactions on Systems, Man and Cybernetics, Vol. 15, No. 4, 1985, pp. 580-585.

[6] W. Yang, H. Yan, J. Wang and J. Yang, "Face Recognition Using Complete Fuzzy LDA,” 19th Conference on International Pattern Recognition, Tampa, 8-11 December 2008, pp. 1-4.

[7] X.-N. Song and Y. Zheng, "A Complete Fuzzy Discriminant Analysis Approach for Face Recognition,” Applied 
Soft Computing, Vol. 10, No. 1, 2010, pp. 208-214.

[8] M.-Y. Shieh, C.-M. Hsieh and J.-Y. Chen, "PCA and LDA-Based Fuzzy Face Recognition System,” SICE Annual Conference, Taipei, 18-21 August 2010, pp. 16101615.

[9] A. Eleyan and H. Demirel, "Face Recognition System Based on PCA and Feedforward Neural Networks," Proceedings of Computational Intelligence and Bioinspired Systems, Barcelona, June 2005, pp. 935-942. doi:10.1007/11494669_115
[10] S. Lawrence, C. L. Giles, A. C. Tsoi and A. D. Back, "Face Recognition: A Convolutional Neural-Network Approach,” IEEE Transactions on Neural Networks, Vol. 8, No. 1, 1997, pp. 98-113. doi:10.1109/72.554195

[11] Z. J. Yu, "Face Recognition with Eigenface,” Proceeding of the IEEE International Conference on Industrial Technology, Guangzhou, 5-9 December1994, pp. 434-438.

[12] ORL, “The Database of Faces,” 2011.

http://www.cl.cam.ac.uk/research/dtg/attarchive/facedatas e.html 